\title{
Remembering Giulio Auriemma (1943-2018) (Friend and Scientist)
}

\section{Franco Giovannelli*}

INAF - Istituto di Astrofisica e Planetologia Spaziali, Via del Fosso del Cavaliere, 100, 00133

Roma, Italy

E-mail: Eranco.giovannellidinaf.it

It is a "hard saying" for me to remember Giulio Auriemma in this context. At these international meetings, he always shone brightly for his astute contributions, derived not only from his profound knowledge of physics, but also from his warmth and humor. I remember him as a great scientist, a fine mentor, and above all as a great friend.

Multifrequency Behaviour of High Energy Cosmic Sources - XIII - MULTIF2019

3-8 June 2019

Palermo, Italy

${ }^{*}$ Speaker. 


\section{Introduction}

Giulio was born in Roma on May 5, 1943 and passed away in Roma on November 22, 2018.

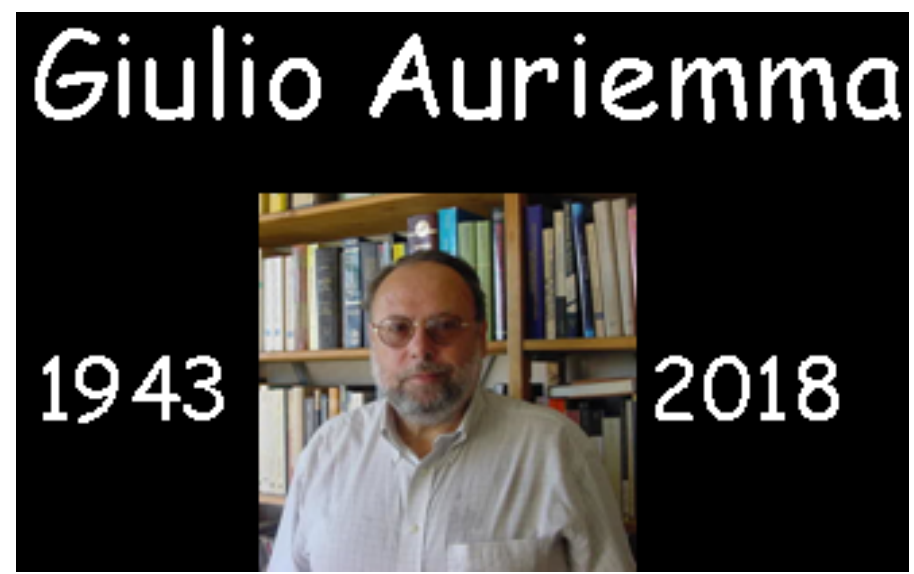

Figure 1: Giulio Auriemma: Scientist.

Giulio's long career as a scientist evolved in his research and teaching activities in several public institutions, including:

- Cattedra di Astrofisica, "La Sapienza" University of Roma, Italy;

- Laboratorio di Astrofisica Spaziale, CNR, Frascati, Italy;

- INFN, Frascati, Italy;

- Università di Basilicata, Potenza, Italy;

- INFN, Roma, Italy;

- CERN, Geneva, Switzerland.

\section{Giulio and Franco at the beginning}

I met Giulio at the beginning of January 1970 in Frascati, Italy, where a group of young scientists and technicians were working under the direction of Livio Gratton, a full professor of Astrophysics at the La Sapienza University of Roma.

This group was brought together via funding from the Italian CNR (Consiglio Nazionale delle Ricerche) in order to prepare a payload for X-ray astronomy to be launched aboard a Skylark rocket. The rocket was made available by the ESA (European Space Agency) from the Woomera launch complex in Australia.

I was involved in a collaboration with Giulio in the design and realization of a fast telemetry system (48 kbit/s was at that time surprisingly fast!) for that experiment (Auriemma \& Giovannelli, 1972).

Three short months later, after an intense effort, headed by Giulio, we founded the "Laboratorio di Astrofisica Spaziale (LAS), CNR". 
We continued to work together, developing new position-sensitive X-ray detectors and associated electronics, thus originating our first publications in international journals.

Meanwhile, Emiliano (Giulio and Minni's son), and Francesca (my and Anna's first daughter) were born in September and November 1970, respectively. They grew up together. Giulio and I were in truth unified by both work and familial ties.

\section{The first scientific results}

Thanks to an idea of Giulio, as noted earlier, we started the development of new positionsensitive soft X-ray detectors and the associated electronics. Thanks to my experience with electronic systems for nuclear detectors, and in particular with charge preamplifiers acquired during my period of work at the Nuclear Center of Casaccia belong to the CNEN (Comitato Nazionale per l'Energia Nucleare) we developed a "simple" and inexpensive charge preamplifier with total dissipation of only $38 \mathrm{~mW}$ and sensitivity of $3.2 \times 10^{-8}$ Volts/electrons for space applications (Auriemma, Giovannelli \& Mengucci, 1973). This was used in association with a position sensitive soft X-ray detector (Auriemma et al., 1974). This preamplifier is flying aboard INTEGRAL, only with the use of a further generation of FET (Field Effect Transistor).

In the meantime, Pietro Ubertini and Enrico Costa (now very well known scientists) joined us as recent graduates in Physics from the "La Sapienza" University of Rome. From that moment on, though our scientific paths were later separated somewhat, Pietro, Enrico and I always considered Giulio our mentor.

During this time, as I noted, we started to develop position-sensitive detectors for X-rays, and we published in 1974 the paper "A position-sensitive semi-proportional chamber for ultra-soft Xrays" in Nuclear Instruments and Methods (Auriemma et al., 1974). This was the first position sensitive soft X-ray detector, and later led to our detectors for hard X-rays (Costa et al., 1978). These latter flew aboard a transoceanic balloon for detecting hard X-ray emission from cosmic sources. This experiment, called HXR76, gave an important result from the Seyfert galaxy NGC 4151 obtained with data collected during the long transocean flight from Milo base (Sicily, Italy) to the eastern coast of the U.S.A. along the 38th parallel (Auriemma et al., 1978).

By the mid-70s, we had clearly come to the realization that in order to understand the physics governing cosmic sources, it would be necessary to take measurements in energy bands as wide as possible. For this reason we started to perform also optical measurements, possibly simultaneous with those in X-ray band. And to become independent we designed and constructed a Simultaneous, fast, double head UBV photometer (Piccioni et al., 1979), and an Ultrafast, simultaneous, four channel photometer (Giovannelli et al., 1980).

Around the end of the 1970's, our scientific interests began to diverge and therefore our paths separated, but not our friendship.

\section{The scientific path of Giulio}

Giulio left the LAS and started to collaborate with INFN. As an example of this new activity I refer to High-energy neutrino emission from binary X-ray sources (Auriemma, 1988), and to ASTROMAG: A particle spectrometer for the space station (Auriemma, Bilokon \& Grillo, 1988). 
Giulio became a Full Professor of Physics at the University of Basilicata in Potenza, and following this, to actively attend our workshops, as demonstrated for instance by the paper Composition of Cosmic rays and Astrophysics (Auriemma, 1993).

In the middle of 1990's our scientific interests converged once more, thanks to my stay as Professor of Physics in his university for one academic year (1989-1990). The result was the study of the feasibility of a ground-based detector via exploitation of the technique of Čerenkov light stereo imaging of air shower produced by discrete cosmic sources of $\gamma$-rays with an energy threshold of $100 \mathrm{GeV}$, angular resolution (HWHM) of $1 \operatorname{arcmin}(1 / 3 \mathrm{mrad})$, and a cosmic ray background rejection of $\geq 99 \%$.

This experiment, named GAMT (Gamma-ray Air-Cherenkov Multiple Telescope), could have carried out a deep survey of the sky with sensitivity of $1 / 100$ of the Crab in 45 hours of exposure (Auriemma et al., 1995, 1996).

Giulio's participation in the MACRO experiment set up in the National Laboratories of the Gran Sasso of INFN was relevant. Some examples of this activity can be found in Ambrosio et al. (1997a,b, 1998, 2001).

Giulio was also interested in Gravitational Waves (GWs) and their connection with GRBs. Sixteen years before the detection of GWs from NS-NS mergers and associated GRB. He published an interesting letter about this problem (Auriemma, 2001), and later he discussed the same problem at the Vulcano Workshop 2002 on "Frontier Objects in Astrophysics and Particle Physics" (Auriemma, 2003a).

Giulio was also keenly interested in the problems of CP and CPT violations and consequences in the Standard Model, and in models of baryogenesis, contributing to the comprehension of the asymmetry between matter and antimatter (Auriemma, 2003b, 2005, 2008).

Of primary importance was his contribution to the $\mathrm{LHCb}$ experiment in Geneva operating in the Large Hadron Collider (LHC).

Giulio, in addition to teaching Physics at the University of Basilicata in Potenza, has always been active collaborating in the cutting edge experiments of INFN and CERN. He published 711 papers, most of them within the MACRO and LHCb collaborations, but his contribution to astrophysics has also been important, with 114 papers of which some examples can be found in Auriemma (2016, 2017), Aaij et al. - The LHCb Collaboration (2017, 2018, 2019), Albicocco et al., 2019). The latter two papers are the last published with the name of Giulio.

Giulio, as professor, never forgot his obligations to his students. His lessons were always exciting and clear. Moreover Giulio used to worry about helping his students by writing lecture notes for them, such as "Introduction to Classical Electrodynamics" in the academic year 2003/2004 (Auriemma, 2003c).

Finally, I would like to recall Giulio's presence at our workshops to demonstrate how much he contributed to their success both from scientific and human point of views. To this end, in Fig. 2, I report the first page of his Final Remarks of the Frascati Workshop 2013 "Multifrequency Behavior of High Energy Cosmic Sources - X" (Auriemma, 2014), which each reader can find in the latter publication and the acknowledgements that fully describe the atmosphere of our workshops. 


\section{Final Remarks}

Giulio Auriemma ${ }^{1,2}$

\footnotetext{
${ }^{1}$ Universitï̈̈̋ degli Studi della Basilicata, Potenza,Italy

${ }^{2}$ INFN Sezione di Roma, Rome, Italy

Corresponding author: Giulio.Auriemma@cern.ch
}

\section{Acknowledgments}

I am sure to interpret the feelings of all the participants to this very successful meeting thanking

- the scientific committee for having assembled such an interesting program;

- all the speakers for their efforts in communicating results and ideas;

- Daniela and Francesco for the precious help given to run smoothly the meeting;

- Valentina, Lisa, Flavia and Alessandro for the good music and readings, that gave us a moment of true ... "felicitas"

- the weather in Mondello not making us to regret of being segregated inside the lecture room;

- and finally Franco ... everybody knows why!

Figure 2: Concluding remarks of the Frascati Workshop 2013 "Multifrequency Behavior of High Energy Cosmic Sources - X", and acknowledgements made by Giulio (Auriemma, 2014).

\section{Photo Gallery}

I would like to conclude this remembrance with a photograph, shown in Fig. 3, taken during the Frascati Workshop 2013, where on the terrace of the Splendid Hotel La Torre, Giulio and Sergio Colafrancesco (who also passed away in 2018) are portrayed in their splendor.

In order to remember Giulio during our happy meetings, full of science and friendship, developed in Vulcano till 2011 and later in Mondello, I have collected some images among the many available, shown in Fig. 4. This is simply a dear memory of the great scientist and dear friend, Giulio.

\section{References}

[1] Aaij, R. et al. (LHCb Collaboration): 2017, PhRvL 119, Issue 23, id. 232001.

[2] Aaij, R. et al. (LHCb Collaboration): 2018, PhRvD 97, Issue 3, id. 032010.

[3] Aaij, R. et al. (LHCb Collaboration): 2019, EPJC 79, Issue 9, article id. 745, 19 pp. 


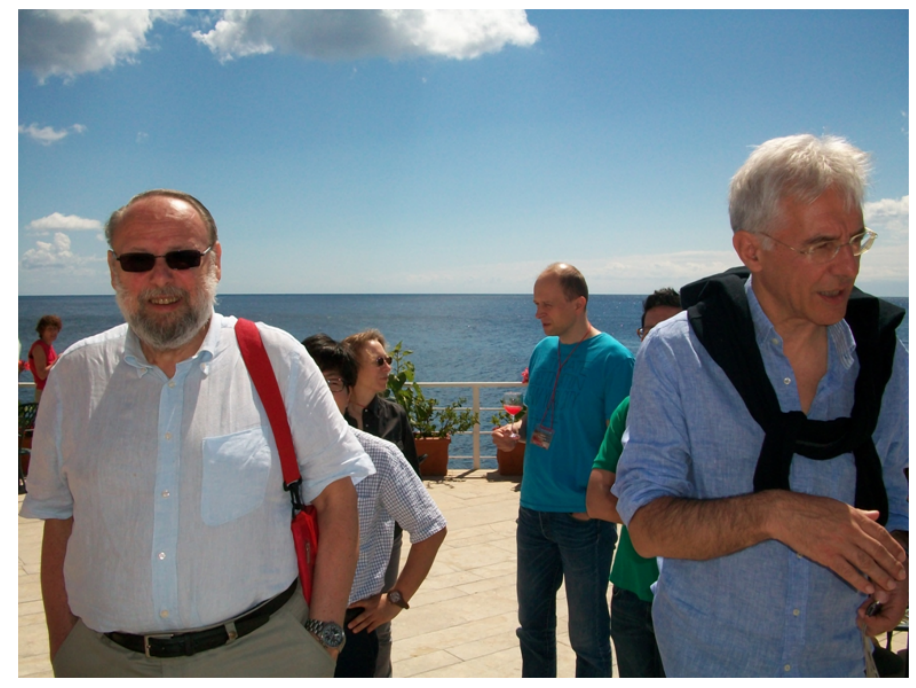

Figure 3: Giulio Auriemma and Sergio Colafrancesco during a coffee break of the Frascati Workshop 2013 on the terrace of the Splendid Hotel La Torre.

[4] Albicocco, F.P., Anderlini, L., Anelli, M., Archilli, F., Auriemma, G., and 53 more: 2019, JInst. 14, Issue 11, P11031.

[5] Ambrosio, M., Antolini, R., Auriemma, G., and 115 more: 1997a, PhRvD 56, Issue 3, 1418-1436.

[6] Ambrosio, M., Antolini, R., Auriemma, G., and 121 more: 1997b, PhLB 406, 249-255.

[7] Ambrosio, M., Antolini, R., Auriemma, G., and 119 more: 1998, APh 8, Issue 3, 123-133.

[8] Ambrosio, M., Antolini, R., Auriemma, G., and 115 more: 2001, ApJ 546, Issue 2, 1038-1054.

[9] Auriemma, G.: 1988, NIMPA 263, Issue 1, 243-248.

[10] Auriemma, G.: 1993, in Frontier Objects in Astrophysics and Particle Physics, F. Giovannelli \& G. Mannocchi (Eds.), SIF, Editrice Compositori, Bologna, Vol. 40, 423-432.

[11] Auriemma, G.: 2001, JPhG 27, Issue 11, L109-L116.

[12] Auriemma, G.: 2003a, in Frontier Objects in Astrophysics and Particle Physics, F. Giovannelli \& G. Mannocchi (Eds.), SIF, Editrice Compositori, Bologna, Vol. 85, 229-251.

[13] Auriemma, G.: 2003b, in Multifrequency Behaviour of High Energy Cosmic Sources - V, F. Giovannelli \& L. Sabau-Graziati (Eds.), ChJA\&A 8, Supplement, 30-42.

[14] Auriemma, G.: 2003c, Introduzione all'Elettrodinamica Classica, online at https://docplayer.it/44489024-Introduzione-all-elettrodinamica-classica.html

[15] Auriemma, G.: 2005, NCimB 120, Issue 6, 603-613.

[16] Auriemma, G.: 2008, in Multifrequency Behaviour of High Energy Cosmic Sources - VII, F. Giovannelli \& L. Sabau-Graziati (Eds.), ChJA\&A 3, Supplement, 33-44.

[17] Auriemma, G.: 2014, Multifrequency Behaviour of High Energy Cosmic Sources - X, F. Giovannelli \& L. Sabau-Graziati (Eds.), Acta Polytechnica CTU Proceedings 1(1), 322-325.

[18] Auriemma, G.: 2016, in Frontier Research in Astrophysics - II, F. Giovannelli \& L. Sabau-Graziati (Eds.), Online at https://pos.sissa.it/cgi-bin/reader/conf.cgi?confid=269, id. 2. 


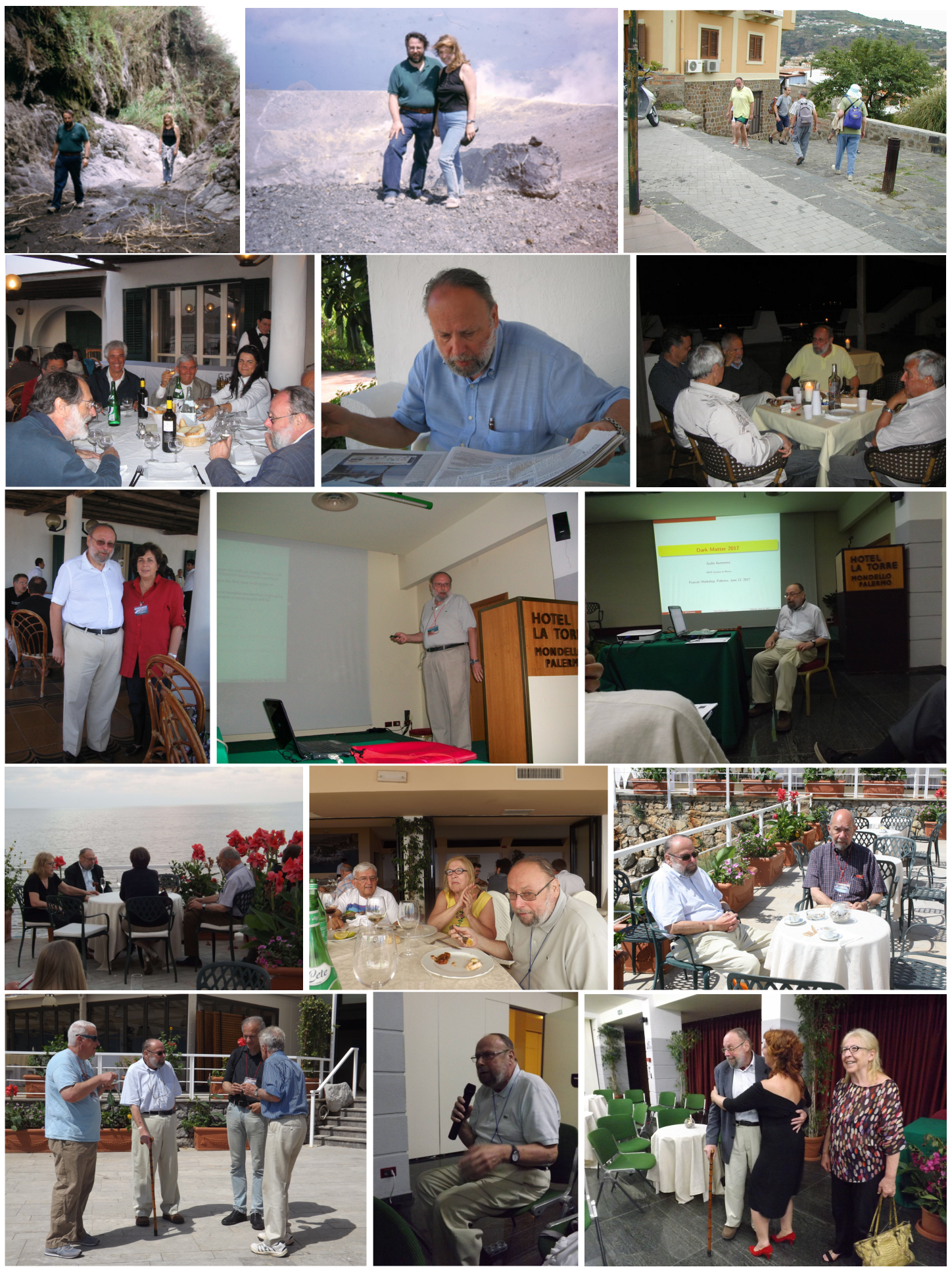

Figure 4: A photo gallery of Giulio from 1984 to 2018 during the many workshops in Vulcano, till 2011 and later in Mondello. 
[19] Auriemma, G.: 2017, Multifrequency Behaviour of High Energy Cosmic Sources - XII, F. Giovannelli \& L. Sabau-Graziati (Eds.), Online at https://pos.sissa.it/cgi-bin/reader/conf.cgi?confid=306, id. 2.

[20] Auriemma, G., Giovannelli, F.: 1972, Internal Report of the Laboratorio di Astrofisica Spaziale, LAS-CNR, No. 34.

[21] Auriemma, G., Giovannelli, F., Mengucci, C.: 1973, NIM 113, 473-475.

[22] Auriemma, G., Giovannelli, F., Mastropietro, M. Ubertini, P.: 1974, NIM 120, 497-500.

[23] Auriemma, G., Angeloni, L., Belli, B.M., Bernardi, A., Cardini, D., Costa, E., Emanuele, A., Giovannelli, F., Ubertini, P.: 1978, ApJ 221, L7-L11.

[24] Auriemma, G., Bilokon, H., Grillo, A.F.: 1986, NCimC, Series 1, vol. 9C, 451-458.

[25] Auriemma, G., Capaccioli, M., Costa, E., Dalì, G., Errico, L., Frenkel, A., Giovannelli, F., Golovkin, S., Kozarenko, E., Kreslo, I., Longo, G., Mancini, D., Martellotti, G., Medvedkov, A., Penso, G., Satriano, C., Scarsi, L., Vittone, A.A.: 1995, APh 3, Issue 3, 215-229.

[26] Auriemma, G., Capaccioli, M., Costa, E., Dali, G., Errico, L., Frenkel, A., Giovannelli, F., Golovkin, S., Kozarenko, E., Kreslo, I., Longo, G., Mancini, D., Martellotti, G., Medvedkov, A., Penso, G., Satriano, C., Scarsi, L., Vittone, A.A.: 1996, in Multifrequency Behaviour of High Energy Cosmic Sources - I, F. Giovannelli \& L. Sabau-Graziati (Eds.), Mem. SAIt. 67, N. 1-2, 619-624.

[27] Costa, E., Auriemma, G., Boccaccini, L., Emanuele, A., Manchanda, R.K., Mastropietro, M., Medici, G., Ubertini, P.: 1978, NIM 156, 57-61.

[28] Giovannelli, F., Auriemma, G., Costa, E., Frutti, M., Mastropietro, M., Pica, F., Piccioni, A.: 1980, AcA 30, N. 4, 565-576.

[29] Piccioni, A., Bartolini, C., Guarnieri, A., Giovannelli, F.: 1979, AcA 29, N. 3, 463-468.

On behalf of all the friends with whom we have shared many participations in our workshops, I would like to greet Giulio with an image that portrays him while formulating one of his astute questions (Fig. 5).

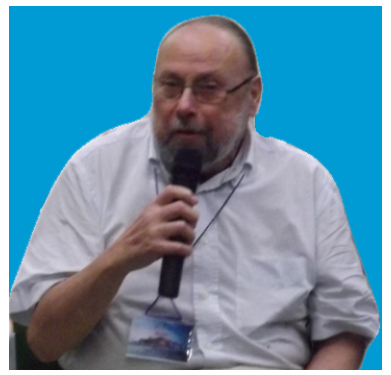

Figure 5: Giulio Auriemma asking a question during the Frascati Workshop 2017.

\section{Dear Giulio rest in peace.}

\section{Acknowledgments}

It is with great pleasure that I want to thank the referee, Dr James Howarth Beall (Jim), for his help in improving the text of this commemoration.

This research has made use of "The NASA's Astrophysics Data System". 